\title{
Higher Curvature Gravity from Entanglement in Conformal Field Theories
}

\author{
Felix M. Haehl and Eliot Hijano \\ Department of Physics and Astronomy, University of British Columbia, \\ 6224 Agricultural Road, Vancouver, British Columbia V6T 1Z1, Canada \\ Onkar Parrikar \\ David Rittenhouse Laboratory, University of Pennsylvania, 209 S. 33rd Street, Philadelphia, Pennsylvania 19104, USA \\ Charles Rabideau \\ David Rittenhouse Laboratory, University of Pennsylvania, 209 S. 33rd Street, Philadelphia, Pennsylvania 19104, USA \\ and Theoretische Natuurkunde, Vrije Universiteit Brussel (VUB), \\ and International Solvay Institutes, Pleinlaan 2, B-1050 Brussels, Belgium
}

(Received 10 January 2018; revised manuscript received 2 April 2018; published 16 May 2018)

\begin{abstract}
By generalizing different recent works to the context of higher curvature gravity, we provide a unifying framework for three related results: (i) If an asymptotically anti-de Sitter (AdS) spacetime computes the entanglement entropies of ball-shaped regions in a conformal field theory using a generalized Ryu-Takayanagi formula up to second order in state deformations around the vacuum, then the spacetime satisfies the correct gravitational equations of motion up to second order around the AdS background. (ii) The holographic dual of entanglement entropy in higher curvature theories of gravity is given by the Wald entropy plus a particular correction term involving extrinsic curvatures. (iii) Conformal field theory relative entropy is dual to gravitational canonical energy (also in higher curvature theories of gravity). Especially for the second point, our novel derivation of this previously known statement does not involve the Euclidean replica trick.
\end{abstract}

DOI: $10.1103 /$ PhysRevLett.120.201602

Introduction.-It has been known for some time that the emergence of a connected spacetime with local dynamics in holographic conformal field theories (CFTs) is intimately related to quantum entanglement [1,2]. If one wishes to make precise the slogan that "spacetime emerges from entanglement," the simplest question one can ask about dynamics is the following: Since graviton and matter field excitations have to be consistent with Einstein's equations, what is the corresponding consistency condition on state perturbations in the language of CFT entanglement?

The energy of the gravitational field is very sensitive to this question. An important observation is that "canonical energy" in Einstein gravity is the bulk quantity dual to "relative entropy" which quantifies the difference in the entanglement structure of two CFT states [3]. At first order in perturbation theory around the vacuum state, the relative entropy indeed satisfies a consistency condition, which is exactly equivalent to the gravity constraint imposed by linearized Einstein equations [4]: the so-called first law of

Published by the American Physical Society under the terms of the Creative Commons Attribution 4.0 International license. Further distribution of this work must maintain attribution to the author(s) and the published article's title, journal citation, and DOI. Funded by SCOAP ${ }^{3}$. entanglement [5]. A similar analysis involving second order generalizations [6] constrains a bulk field perturbation to satisfy nonlinear Einstein equations [7]. Interestingly, this derivation does not assume the CFT to be holographic. The reason is that second order graviton propagation is secretly still kinematic in the sense that it is almost universal among all covariant theories of gravity: it only depends on two parameters, which correspond to $a$ - and $c$-type central charges of the CFT. To some extent in perturbation theory the emergence of a bulk geometry and Einstein's equations is thus a feature of just the entanglement structure of the CFT state, without reference to further anti-de Sitter (AdS)/CFT assumptions such as regarding the spectrum of the theory.

At the nonlinear level, Einstein's equations have been derived from state perturbations under the assumption of equal $a$ - and $c$-type central charges, which is consistent with a description in terms of Einstein gravity. In this Letter we aim to provide a generalization to higher curvature theories of gravity and thus for completely generic CFTs. This brings about a further interesting subtlety: while in Einstein gravity the holographic computation of CFT entanglement entropy involves evaluating the area of an appropriate extremal surface [8,9], the entanglement entropy functional to be evaluated in higher curvature theories of gravity is more complicated [10,11]. 
Determining this functional correctly, therefore, belongs to the same circle of ideas. By systematically studying higher curvature theories of gravity, we aim to provide a unifying framework for these issues. To achieve this conceptual goal, we need to overcome a technical challenge which consists of generalizing the recent CFT calculation of relative entropy [7] to higher curvature theories of gravity.

Setup.-We consider CFT states $\left|\psi_{\lambda}(\varepsilon)\right\rangle$ that are created by sourcing the stress tensor in the Euclidean path integral over the half plane:

$$
\left\langle\varphi_{(0)} \mid \psi_{\lambda}(\varepsilon)\right\rangle=\int^{\varphi_{(0)}} D \varphi e^{-\int_{-\infty}^{0} d^{d} x_{E}\left(\mathcal{L}_{\mathrm{CFT}}+\varepsilon \lambda_{\mu \nu} T^{\mu \nu}\right)},
$$

where $\varphi$ collectively denotes elementary fields in the CFT with the boundary condition $\varphi(0, \mathbf{x})=\varphi_{(0)}(\mathbf{x})$ at Euclidean time $x_{E}^{0}=0$. For small $\varepsilon$ this leads to a perturbation theory in the deformation of the vacuum state. The reduced density matrix of the region $A$ takes the form $\rho_{A}=\rho_{A}^{(0)}+\varepsilon \delta \rho_{A}+\mathcal{O}\left(\varepsilon^{2}\right)$, where $\rho_{A}^{(0)}$ is the reduced density matrix in the vacuum. Note that we could similarly turn on sources for other primary operators $\mathcal{O}_{\alpha}$ in the path integral. For notational simplicity we will not do so here, but all our calculations work the same way for other primary operators [12].

In holographic theories, this state deformation is dual to coherent excitations of the bulk gravitons. The state perturbations in the CFT then translate to a gravitational perturbation theory of the form

$$
g=g_{\text {AdS }}^{(0)}+\varepsilon \delta g^{(1)}+\varepsilon^{2} \delta g^{(2)}+\ldots .
$$

The Einstein equations, $E^{a b}-\frac{1}{2} T^{a b}=0$, can be expanded in a similar way; $\delta^{(2)} T^{a b}$ will be quadratic in $\delta g^{(1)}$ and therefore allows us to study gravitational backreaction.

Our calculations will only be sensitive to two characteristics of the CFT: its $a$ - and $c$-type central charges. We define these in general as the normalization of the universal part of ball entanglement entropy in vacuum, and the normalization of the universal stress tensor two-point function, respectively. In four-dimensional CFTs, these can be recognized as the coefficients in the conformal anomaly. The $a$-type central charge sets the AdS scale for $g_{\text {AdS }}^{(0)}$ above in the usual way.

Wald formalism and beyond.-Consider a covariant theory of gravity governed by the Lagrangian $(d+1)$-form

$$
\mathbf{L} \equiv \mathcal{L} \text { vol }, \quad \mathcal{L}=\frac{1}{16 \pi G_{N}}\left(R+\frac{d(d-1)}{\ell^{2}}+f(\text { Riem })\right),
$$

where $f($ Riem) is a function of Riemann tensors, contracted with the metric. We can define the equation of motion form $\mathbf{E}_{\mathbf{L}}$, the presymplectic potential $\boldsymbol{\theta}_{\mathbf{L}}$, and the symplectic current $\omega_{\mathrm{L}}$ as

$$
\begin{aligned}
\delta \mathbf{L} & =-\mathbf{E}_{\mathbf{L}} \cdot \delta g-d \boldsymbol{\theta}_{\mathbf{L}}(\delta g), \\
\boldsymbol{\omega}_{\mathbf{L}}\left(\delta_{1} g, \delta_{2} g\right) & \equiv \delta_{1} \boldsymbol{\theta}_{\mathbf{L}}\left(\delta_{2} g\right)-\delta_{2} \boldsymbol{\theta}_{\mathbf{L}}\left(\delta_{1} g\right) .
\end{aligned}
$$

Noether's theorem can be expressed as an off-shell identity:

$$
\omega_{\mathbf{L}}\left(\delta g, \mathfrak{£}_{X} g\right)-\mathcal{G}_{\mathbf{L}}(\delta g, X)=d \chi_{\mathbf{L}}(\delta g, X),
$$

where $X$ is an arbitrary vector field and $\mathcal{G}_{\mathbf{L}}$ is proportional to the equation of motion for $\delta g$. Finally, $\chi_{\mathrm{L}}$ can be defined in terms of the Noether charge, but we only require the property that its integral defines notions of modular energy and entropy. Specifically, integrating $d \chi_{\mathbf{L}}$ over a spacelike slice $\Sigma_{A}$ of the AdS-Rinder wedge, one finds the following boundary terms:

$$
\begin{aligned}
\int_{A} \chi_{\mathbf{L}}\left(\delta g, \xi_{A}\right) & =\int_{A} d \Sigma^{\mu} \delta T_{\mu \nu}^{\mathrm{grav}} \zeta_{A}^{\nu} \equiv \delta E_{A}^{\mathrm{grav}} \\
\int_{\tilde{A}} \chi_{\mathbf{L}}\left(\delta g, \xi_{A}\right) & =8 \pi \delta \int_{\tilde{A}} \sqrt{\bar{g}} \frac{\partial \mathcal{L}}{\partial R_{a b c d}} n_{a b} n_{c d} \equiv \delta S_{A}^{\text {Wald }},
\end{aligned}
$$

where $\xi_{A}$ is the Killing vector that generates Rindler boosts, $n_{a b}$ is the binormal of the horizon, and $\delta T_{\mu \nu}^{\text {grav }}$ is the holographic stress tensor, which coincides with $\delta\left\langle T_{\mu \nu}^{\mathrm{CFT}}\right\rangle$ [4]. At this linear order in perturbation theory, both quantities are proportional to the ones in Einstein gravity (the proportionality constant computes the $c$-type central charge [13]). Their sum can be interpreted as the Hamiltonian associated with $\xi_{A}$ evolution [14].

Second order perturbations: For Einstein gravity [i.e., $f($ Riem $)=0$ ] Hollands and Wald [6] have shown that there exists a gauge where the off-shell identity, Eq. (5), holds at any order in $\varepsilon$. Working in Gaussian null coordinates where the future- and past-directed null normal vectors to the unperturbed extremal surface are $\partial_{\ell^{+}}$and $\partial_{\ell^{-}}$, this gauge condition amounts to imposing (i) the Killing vector $\xi_{A}=$ $2 \pi\left(\ell^{+} \partial_{\ell_{+}}-\ell^{-} \partial_{\ell^{-}}\right)$remains Killing near the surface, and (ii) the coordinate location of the surface, defined by extremization of the entanglement entropy functional, is fixed:
(i) $0=\left.£_{\xi_{A}} g\right|_{\ell^{+}, \ell^{-}=0}$,
(ii) $0=\left.K_{\alpha \beta}^{ \pm} \frac{\delta S_{A}^{\text {Wald }}}{\delta \bar{g}_{\alpha \beta}}\right|_{\ell^{+}, \ell^{-}=0}$.

For a perturbation $\delta g^{(1)}=\gamma$ in this gauge, we have the following generalization of the second order identity of [6]:

$\delta^{(2)}\left[E_{A}^{\text {grav }}-S_{A}^{\text {Wald }}\right]=\int_{\Sigma_{A}} \omega_{\mathbf{L}}\left(\gamma, £_{\xi_{A}} \gamma\right)-\int_{\Sigma_{A}} \delta^{(2)} \mathcal{G}_{\mathbf{L}}$,

where $\delta^{(2)} \mathcal{G}_{\mathbf{L}}$ vanishes if and only if $\delta g^{(2)}$ satisfies the gravitational equations of motion associated with $\mathbf{L}$ at $\mathcal{O}\left(\varepsilon^{2}\right)$. In the higher curvature gravity context, a gauge of the form Eq. (7) (such that Eq. (8) holds) exists in general. We explicitly demonstrate this for curvature squared 
theories by solving the corresponding inhomogeneous differential equation. The condition involving the Killing vector $\xi_{A}$ does not change, while the location of the surface should be defined by extremization of the promoted entanglement entropy functional $[10,15]$. Equation (8) is the central gravitational identity that we use.

Entanglement entropy functional: The functional $S_{A}^{\text {Wald }}$ of Eq. (6) is a good definition of entropy for stationary Killing horizons, where ambiguities in the definition of the Noether charge are irrelevant [16]. However, in a higher curvature theory of gravity and at second order in perturbation theory around AdS spacetime, it is not the correct functional for computing entanglement entropy.

The Noether charge ambiguity in the definition of Wald entropy can be described according to Jacobson, Kang, and Myers (JKM) $[17,18]$. This JKM ambiguity can be written as a $(d-1)$-form $\boldsymbol{Y}$, which shifts the quantities entering Noether's theorem as follows $[17,19]$ :

$$
\begin{aligned}
\omega_{\mathbf{L}}\left(\delta_{1} g, \delta_{2} g\right) & \rightarrow \omega_{\mathbf{L}}+d\left[\delta_{1} \boldsymbol{Y}\left(\delta_{2} g\right)-\delta_{2} \boldsymbol{Y}\left(\delta_{1} g\right)\right] \\
\chi_{\mathbf{L}}\left(\delta g, \xi_{A}\right) & \rightarrow \chi_{\mathbf{L}}+\delta \boldsymbol{Y}\left(£_{\xi_{A}} g\right)-\xi_{A} \cdot d \boldsymbol{Y}(\delta g) .
\end{aligned}
$$

Note that this redefinition leaves Eqs. (5) and (8) invariant.

The two extrinsic curvatures $K_{\alpha \beta}^{ \pm}$of $\tilde{A}$ have boost weights $\mp 1$ (i.e., $£_{\xi_{A}} K_{\alpha \beta}^{ \pm}=\mp K_{\alpha \beta}^{ \pm}$). Since our second order approach is only sensitive to expressions quadratic in the extrinsic curvatures, the second order JKM ambiguity has to be of the form $\boldsymbol{Y}(\delta g)=\boldsymbol{Y}_{(E E)} \equiv \frac{1}{2} \boldsymbol{\epsilon} \delta\left(\mathfrak{B}_{\alpha \beta}^{\gamma \delta} K^{+\alpha \beta} K_{\gamma \delta}^{-}\right)$, where $\mathfrak{B}_{\alpha \beta}^{\gamma \delta}$ is some boost-invariant tensor built out of the metric (and its derivatives). Figuring out the correct entanglement entropy functional in higher derivative theories of gravity to $\mathcal{O}\left(K^{2}\right)$, is equivalent to determining the coefficient tensor $\mathfrak{B}_{\alpha \beta}^{\gamma \delta}$ in terms of the gravitational Lagrangian.

Once the ambiguity has been fixed, $S_{A}^{\text {Wald }}$ appearing in Eqs. (6), (7), and (8) should be replaced with $S_{A}^{E E}$.

Fixing the JKM ambiguity: We wish to highlight two recent approaches to fixing this ambiguity.

(1) A formula for $\mathfrak{B}_{\alpha \beta}^{\gamma \delta}$ in terms of the Lagrangian can be derived using Euclidean methods and the bulk replica trick [20] and demanding that entanglement entropy be computed correctly $[10,11]$. One finds (after analytic continuation) [21]

$$
S_{A}^{E E}=S_{A}^{\text {Wald }}+4 \pi \int_{\tilde{A}} \sqrt{\bar{g}} \frac{\partial^{2} \mathcal{L}}{\partial R_{+\alpha+\beta} \partial R_{-\gamma-\delta}} K_{\alpha \beta}^{+} K_{\gamma \delta}^{-} .
$$

(2) At second order in extrinsic curvatures, the same functional is obtained by demanding that it satisfies a linearized second law for compact horizons [23].

In this Letter, we will derive the solution Eq. (10) using a third, and completely Lorentzian method. We will not use the replica trick, but instead employ a direct calculation of CFT entanglement entropy which is a generalization of the methods recently developed in Ref. [7] (see also Refs. [24-26]).

Note that we only work with states which are second order in perturbation theory around AdS spacetime. Furthermore, the region $A$ for us will be ball shaped. These are small drawbacks from the general situation (in particular, we will not encounter the "splitting problem" $[27,28]$ that renders the method of Ref. [10] ambiguous at higher order in extrinsic curvatures), we also gain something new compared to Refs. [10,11], in addition to not relying on the Euclidean replica trick: We do not need to assume large central charge or any other aspects of holography. Our results constrain a subsector of holography which is completely universal and applies to all CFTs: We will construct an entanglement entropy functional, which computes second order entanglement entropy from an auxiliary geometry for any CFT.

Canonical energy: We define the canonical energy in $f$ (Riem) theories of gravity the same way as in Einstein gravity (as an integral over the form $\omega_{\mathbf{L}}$ ), but with a fixed choice of ambiguity $\mathbf{Y}$ in terms of extrinsic curvatures, thus generalizing [6]

$$
W_{\Sigma_{A}}\left(\gamma, £_{\xi_{A}} \gamma\right) \equiv \delta^{(2)}\left[E_{A}^{\text {grav }}-S_{A}^{E E}\right] .
$$

This is analogous to Eq. (8) after fixing the ambiguity $\boldsymbol{Y}$ in a nontrivial way as above. We will show that the canonical energy $W_{\Sigma_{A}}$ is the quantity dual to relative entropy in the CFT.

Relative entropy: From CFT to AdS.-The third ingredient for our argument is an explicit calculation of relative entropy at second order in the state perturbation Eq. (1):

$\left.\delta^{(2)} S\left(\rho_{A} \| \rho_{A}^{(0)}\right) \equiv \frac{d^{2}}{d \varepsilon^{2}} \operatorname{Tr}\left(\rho_{A} \log \rho_{A}-\rho_{A} \log \rho_{A}^{(0)}\right)\right|_{\varepsilon=0}$.

This quantity can only depend on the two-point function of the stress tensor used to create the state. Indeed, from Eq. (1) it is straightforward to show that the first order perturbation of the density matrix takes the form $\delta \rho_{A}=\rho_{A}^{(0)} \int \lambda_{\mu \nu} T^{\mu \nu}$, and $\delta^{(2)} S\left(\rho_{A} \| \rho_{A}^{(0)}\right)$ is quadratic in $\delta \rho_{A}$. A more careful integral expansion of the logarithm shows

$$
\begin{aligned}
\delta^{(2)} S\left(\rho_{A} \| \rho_{A}^{(0)}\right)= & -\int d \tau_{a} d Y_{a} \tilde{\lambda}_{\mu \nu}\left(\tau_{a}, Y_{a}\right) \\
& \times \int d \tau_{b} d Y_{b} \tilde{\lambda}_{\rho \sigma}\left(\tau_{b}, Y_{b}\right) \\
& \times \int_{-\infty}^{\infty} \frac{d s}{16 \sinh ^{2}\left(\frac{s+i \epsilon \operatorname{sgn}\left(\tau_{a b}\right)}{2}\right)} \\
& \times\left\langle T^{\mu \nu}\left(\tau_{a}, Y_{a}\right) T^{\rho \sigma}\left(\tau_{b}+i s, Y_{b}\right)\right\rangle,
\end{aligned}
$$

where the first two integrals are over the Euclidean space $S^{1} \times \mathbb{U}^{d-1}$ with $\tau_{a b} \equiv \tau_{a}-\tau_{b}$ being the difference of 
Euclidean times. The sources $\tilde{\lambda}_{\mu \nu}(\tau, Y) \equiv \lambda_{\mu \nu}(\tau, Y) \Omega^{-2}(\tau, Y)$ involve a suitable conformal factor. The parameter $s$ can be thought of as (real) modular time for evolution with the modular Hamiltonian $H_{A}=-\log \rho_{A}^{(0)}$.

Importantly, the two-point function in Eq. (13) can be represented as the asymptotic symplectic flux in an auxiliary AdS geometry. This was pointed out in Ref. [7] for Einstein gravity. In the case of a more general gravitational Lagrangian $\mathbf{L}$ a systematic procedure can be followed, which is outlined in the Supplemental Material [29]. The result takes the same form as for Einstein gravity,

$\left\langle T^{\rho \sigma}\left(\tau_{a}, Y_{a}\right) T^{\mu \nu}\left(\tau_{b}+i s, Y_{b}\right)\right\rangle=-\frac{C_{T}}{C_{T}^{\text {grav }}} \int_{r_{B} \rightarrow \infty} d s_{B} d Y_{B} \omega_{\mathbf{L}}\left(K_{E, \mathbf{L}}^{\rho \sigma}\left(i s_{B}, r_{B}, Y_{B} \mid \tau_{a b}, Y_{a}\right), K_{R, \mathbf{L}}^{\mu \nu}\left(s_{B}, r_{B}, Y_{B} \mid s, Y_{b}\right)\right)$,

where now $\mathbf{L}$ is a gravitational Lagrangian of our choice, and $K_{E, \mathbf{L}}$ and $K_{R, \mathbf{L}}$ are Euclidean and retarded bulkboundary propagators [30]. The gravitational computation reproduces the stress tensor correlator with a normalization $C_{T}^{\text {grav }}$ fixed by the chosen gravitational Lagrangian (see Supplemental Material [31] for an explicit expression in terms of curvature squared couplings). Since the form of two-point functions of the stress tensor is universal, the correct CFT correlation function can be reproduced by any gravitational Lagrangian as long as we rescale the final answer as in Eq. (14) so as to fix the normalization.

In order to match the CFT result for relative entropy onto the gravitational identity, the Lagrangian should be chosen such that, on the one hand, $C_{T}^{\text {grav }}=C_{T}$. Because of the universality of the two-point function of the stress tensor, $\delta^{(2)} S\left(\rho_{A} \| \rho_{A}^{(0)}\right)$ is only sensitive to the gravitational Lagrangian through this parameter. Of course, if we have a valid holographic dual we could use its bulk Lagrangian, but we wish to consider the more general possibility of constructing an auxiliary bulk theory for any CFT.

On the other hand, the entanglement entropy for ballshaped regions in the vacuum is universal up to a normalization $a_{*}$ [32-36]. In order that the background geometry $g_{\text {AdS }}^{(0)}$ correctly reproduces this normalization we must require that $a_{*}^{\text {grav }}=a_{*}$ (this fixes the parameter $\ell_{\text {AdS }}$ of our auxiliary geometry). See again the Supplemental Material [31] for explicit expressions.

In Ref. [7],

$$
a_{*}^{\text {grav (Einstein) }}=\frac{(d-1) \pi^{d}}{\Gamma(d+2)} C_{T}^{\text {grav (Einstein) }},
$$

was used for Einstein gravity, so that the two matching conditions above imposed a CFT constraint between $C_{T}$ and $a_{*}$. For general theories of gravity Eq. (15) need not hold, so we can relax our assumptions. Instead, suitable choices of the parameters can always be found to match the $C_{T}$ and $a_{*}$ given by the CFT.

Modular integral: We now substitute Eq. (14) in Eq. (13) for the relative entropy, and perform the integration over modular time $s$. In Ref. [7] this integration was performed carefully for Einstein gravity. The details of this integral are the same in the present case, and so we merely state the result:

$$
\begin{aligned}
\delta^{(2)} S\left(\rho \| \rho_{A}^{(0)}\right)= & \int_{\Sigma_{A}} \omega_{\mathbf{L}}\left(h, \mathcal{L}_{\xi_{A}} h\right) \\
& -\int_{\mathcal{H}^{+}} \omega_{\mathbf{L}}\left(h, I^{(+)}\right)-\int_{\mathcal{H}^{-}} \omega_{\mathbf{L}}\left(h, I^{(-)}\right),
\end{aligned}
$$

where $\mathcal{H}^{ \pm}$are the future and past horizons of the AdS-Rindler wedge, and

$$
\begin{aligned}
h_{m n}\left(\ell^{+}, \ell^{-}, Y_{B}\right)= & \frac{1}{2} \int d \tau d Y \lambda_{\mu \nu}(\tau, Y) \Omega^{-2}(\tau, Y) \\
& \times K_{E ; m n}^{\mu \nu}\left(\ell^{+}, \ell^{-}, Y_{B} \mid \tau, Y\right)
\end{aligned}
$$

is the bulk graviton sourced by the (Euclidean) boundary source. Further, $I^{(+)}$can be conveniently expressed in terms of the contour integral

$$
\begin{aligned}
I_{m n}^{(+)}= & i \lim _{\ell^{-} \rightarrow 0} \oint_{|w|=1-\epsilon} d w \frac{e^{-s_{*}^{+}}}{\left(w-e^{-s_{*}^{+}}\right)^{2}} J^{p}{ }_{m} J^{q}{ }_{n} \\
& \times h_{p q}\left(\frac{\ell^{+}}{w e^{s_{*}^{+}}}, \ell^{-} w e^{s_{*}^{+}}, Y_{B}\right),
\end{aligned}
$$

where $J^{a}{ }_{b}$ is the Jacobian matrix corresponding to the boost $\ell^{ \pm} \rightarrow\left(w e^{s_{*}^{+}}\right)^{\mp 1} \ell^{ \pm}$, and similarly for $I_{m n}^{(-)}$. By expanding $h_{p q}$ around the extremal surface $\ell^{ \pm}=0$, we can easily perform the above contour integration and obtain $I_{m n}^{( \pm)}$in an expansion around the extremal surface (see Ref. [7] for details). It is crucial to note that the metric perturbation $h_{m n}$ is in a particular gauge, namely, the generalized de-Donder gauge (and not the Hollands-Wald gauge which was discussed previously). It is the nice analytic structure of the propagator in this gauge which allows us to perform the modular $s$ integral straightforwardly.

The final step in the calculation then, is to reexpress the result Eq. (16) in the Hollands-Wald gauge. This is conceptually again similar to the Einstein gravity case, but in practice it is significantly more complicated. This calculation is the main new technical result of this Letter, 
and can be found in the Supplemental Material [37]. The final answer is

$$
\begin{aligned}
\delta^{(2)} S\left(\rho \| \rho_{A}^{(0)}\right)= & \int_{\Sigma_{A}} \omega_{\mathbf{L}}\left(g, \gamma, \mathcal{L}_{\xi_{A}} \gamma\right) \\
& +4 \pi \int_{\tilde{A}} \sqrt{\bar{g}} \frac{\partial^{2} \mathcal{L}}{\partial R_{+\alpha+\beta} \partial R_{-\gamma-\delta}} \delta K_{\alpha \beta}^{+} \delta K_{\gamma \delta}^{-},
\end{aligned}
$$

where $\gamma=h+\mathfrak{f}_{V} g$ is the gauge transformed perturbation such that $\gamma$ satisfies the Hollands-Wald gauge conditions Eq. (7), and the second line gives extrinsic curvature contributions which were not present in Einstein gravity. Note that Eq. (19) is an entirely field theoretic equationthe right-hand side looks like gravity, but the bulk geometry is a priori auxiliary.

Results.-Having established the central Eqs. (8), (11), and (19), we are now ready to draw some conclusions. We can solve this set of equations either for the entanglement entropy functional, or for the equations of motion, or for the canonical energy, in each case generalizing previous results, using new (Lorentzian) techniques. Taken together, these results provide a unifying framework for various ideas discussed hitherto. We now describe these three points of view. Figure 1 provides a summary of these ideas.

Solving for the entropy functional: We can rederive the results of $[10,11]$ without any reference to Euclidean methods such as the replica trick, by solving our basic equations for the entanglement entropy functional $\delta^{(2)} S_{A}^{E E}$. Assuming (i) the equality of canonical energy Eq. (11) and boundary relative entropy Eq. (19), and (ii) the second order gravitational equations of motion (i.e., $\delta^{(2)} \mathcal{G}_{\mathbf{L}}=0$ ), we find

$$
\begin{aligned}
\delta^{(2)} S_{A}^{E E}= & \delta^{(2)} S_{A}^{\text {Wald }} \\
& -4 \pi \int_{\tilde{A}} \sqrt{\bar{g}} \frac{\partial^{2} \mathcal{L}}{\partial R_{+\alpha+\beta} \partial R_{-\gamma-\delta}} \delta K_{\alpha \beta}^{+} \delta K_{\gamma \delta}^{-} .
\end{aligned}
$$

This is consistent with previous results on the holographic entanglement entropy.

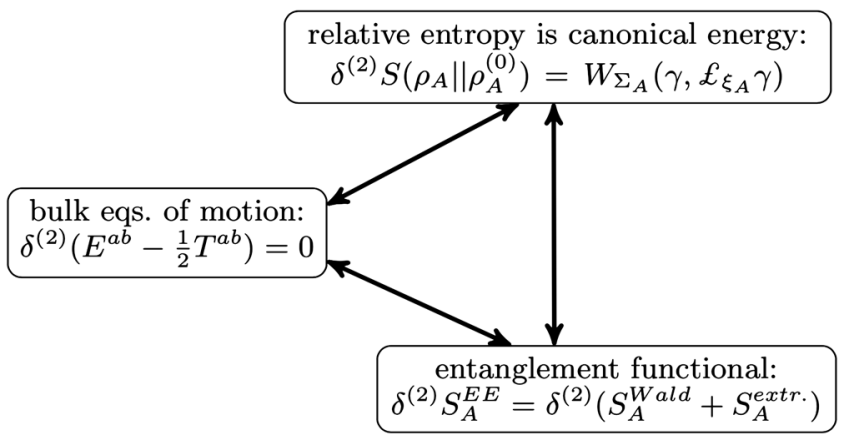

FIG. 1. Three concepts of interest here. We generalize all of them for higher curvature theories of gravity and show that assuming any two of them implies the third.
Solving for the equations of motion: Assuming that (i) we already knew the correct entanglement entropy functional (for instance, from Refs. [10,11]), and (ii) that again the relative entropies Eqs. (11) and (19) match, we could similarly solve for the integrated equations of motion:

$$
\int_{\Sigma_{A}} \delta^{(2)} \mathcal{G}_{\mathbf{L}}=0
$$

Since this holds for all ball-shaped regions (including boosted ones), one concludes $\mathcal{G}_{\mathbf{L}}=0$ and the equations of motion to second order in perturbation theory hold locally. This is a generalization of Ref. [7] to the case of CFTs without any assumption about equality of $a$ - and $c$-type central charges. Indeed, for any value of the central charges (such that $(a-c) / c$ is small), we can find many different bulk Lagrangians reproducing these values, and derive the associated second order equations of motion.

Solving for canonical energy: Finally, we note that assuming (i) the correct entanglement entropy functional, and (ii) the second order equations of motion, the results Eqs. (8), (11), and (19) give a generalization of Ref. [3]: we find explicitly that

$$
W_{\Sigma_{A}}\left(\gamma, £_{\xi_{A}} \gamma\right)=\delta^{(2)} S\left(\rho_{A} \| \rho_{A}^{(0)}\right)
$$

i.e., the equality of bulk canonical energy and boundary relative entropies defined in Eqs. (11) and (12). The new aspect with higher curvature couplings is the extrinsic curvature contributions to canonical energy, i.e., a particular fixing of the $\mathbf{Y}$ ambiguity in the Noether charge formalism. We have thus identified the appropriate notion of gravitational canonical energy in this scenario.

Conclusion.-By studying higher curvature theories of gravity, we were able to lift some ambiguities and drop some assumptions in the context of discussions revolving around notions of energy and entropy in gravity and CFTs. This allowed us to provide a unifying framework for understanding the intimate relations between CFT relative entropy, gravitational equations of motion, and the fixing of ambiguities in the definition of canonical energy and entanglement entropy functionals appropriate for general theories of gravity.

Our analysis is perturbative in nature, dealing with second order deformations of the CFT vacuum state (or field excitations on empty AdS spacetime). It would be very interesting to see if the circle of ideas presented here extends to higher orders in perturbation theory. One expects that already at third order, the CFT calculation will involve three-point functions. By sourcing arbitrary primary operators in the Euclidean path integral this would allow us to access the operator product expansion, and hence provide more detailed constraints based on the requirement of a geometric entropy formula. We leave this interesting question for the future. 
We thank Tom Faulkner and Mark Van Raamsdonk for initial collaboration and helpful discussions. We also thank Joan Camps, Ted Jacobson, Aitor Lewkowycz, Rob Myers, Jonathan Oppenheim, and Antony Speranza for helpful discussions. All of us wish to acknowledge support from the Simons Foundation through the It-from-Qubit collaboration. C. R. was also supported by the Belgian Federal Science Policy Office, by FWO-Vlaanderen, and by Vrije Universiteit Brussel.

[1] M. Van Raamsdonk, arXiv:0907.2939.

[2] J. Maldacena and L. Susskind, Fortschr. Phys. 61, 781 (2013).

[3] N. Lashkari and M. Van Raamsdonk, J. High Energy Phys. 04 (2016) 153.

[4] T. Faulkner, M. Guica, T. Hartman, R. C. Myers, and M. Van Raamsdonk, J. High Energy Phys. 03 (2014) 051.

[5] J. Bhattacharya, M. Nozaki, T. Takayanagi, and T. Ugajin, Phys. Rev. Lett. 110, 091602 (2013).

[6] S. Hollands and R. M. Wald, Commun. Math. Phys. 321, 629 (2013).

[7] T. Faulkner, F. M. Haehl, E. Hijano, O. Parrikar, C. Rabideau, and M. Van Raamsdonk, J. High Energy Phys. 08 (2017) 057.

[8] S. Ryu and T. Takayanagi, Phys. Rev. Lett. 96, 181602 (2006).

[9] V. E. Hubeny, M. Rangamani, and T. Takayanagi, J. High Energy Phys. 07 (2007) 062.

[10] X. Dong, J. High Energy Phys. 01 (2014) 044.

[11] J. Camps, J. High Energy Phys. 03 (2014) 070.

[12] In fact for scalar operators, calculations are simpler since there is no bulk gauge redundancy [7].

[13] F. M. Haehl, J. High Energy Phys. 10 (2015) 159.

[14] N. Lashkari, J. Lin, H. Ooguri, B. Stoica, and M. Van Raamsdonk, Prog. Theor. Exp. Phys. 2016, 12 C109 (2016).

[15] X. Dong and A. Lewkowycz, J. High Energy Phys. 01 (2018) 081.

[16] R. M. Wald, Phys. Rev. D 48, R3427 (1993).
[17] V. Iyer and R. M. Wald, Phys. Rev. D 50, 846 (1994).

[18] T. Jacobson, G. Kang, and R. C. Myers, Phys. Rev. D 49, 6587 (1994).

[19] P. Bueno, P. A. Cano, V. S. Min, and M. R. Visser, Phys. Rev. D 95, 044010 (2017).

[20] A. Lewkowycz and J. Maldacena, J. High Energy Phys. 08 (2013) 090.

[21] For $f\left(R, R_{a b}\right)$ theories of gravity, see also Ref. [22].

[22] M. R. Mohammadi Mozaffar, A. Mollabashi, M. M. SheikhJabbari, and M. H. Vahidinia, Phys. Rev. D 94, 046002 (2016).

[23] A. C. Wall, Int. J. Mod. Phys. D 24, 1544014 (2015).

[24] T. Faulkner, J. High Energy Phys. 05 (2015) 033.

[25] T. Faulkner, R. G. Leigh, and O. Parrikar, J. High Energy Phys. 04 (2016) 088.

[26] G. Sarosi and T. Ugajin, J. High Energy Phys. 01 (2018) 012.

[27] A. Bhattacharyya and M. Sharma, J. High Energy Phys. 10 (2014) 130

[28] R.-X. Miao and W.-z. Guo, J. High Energy Phys. 08 (2015) 031.

[29] See Supplemental Material III at http://link.aps.org/ supplemental/10.1103/PhysRevLett.120.201602 for twopoint functions as symplectic flux.

[30] D. Marolf, J. High Energy Phys. 05 (2005) 042.

[31] See Supplemental Material II at http://link.aps.org/ supplemental/10.1103/PhysRevLett.120.201602 for a- and $c$-type central charges in curvature squared theories.

[32] S. Ryu and T. Takayanagi, J. High Energy Phys. 08 (2006) 045.

[33] S. N. Solodukhin, Phys. Lett. B 665, 305 (2008).

[34] R. C. Myers and A. Sinha, Phys. Rev. D 82, 046006 (2010).

[35] H. Casini, M. Huerta, and R. C. Myers, J. High Energy Phys. 05 (2011) 036.

[36] L.-Y. Hung, R. C. Myers, and M. Smolkin, J. High Energy Phys. 04 (2011) 025.

[37] Supplemental Material I at http://link.aps.org/supplemental/ 10.1103/PhysRevLett.120.201602 for derivation of the central CFT result. 